\title{
ÖKOLOGIE UND GESELLSCHAFT: ÜBER ETHIK UND RATIONALITÄT IM VERHÄLTNIS VON SYSTEM UND UMWELT
}

\begin{abstract}
Ohne einen erweiterten Ansatz unseres Denkens und Handelns wird es keine Lösung der Umweltproblematik der Moderne geben. Vernunft und Ethik sind daher gefordert, die Möglichkeiten unserer Einsicht in diese Problematik und unserer Orientierung ihr gegenüber auszubauen. Um die prinzipiellen Schwierigkeiten zu verdeutlichen, die auf diesem Wege zu bewältigen sind, befasst sich der Beitrag mit den Themen „Selbstreferenz und Umweltethik“, „Azentrizität und ökologische Vernunft“ und dem Verhältnis von „Ökonomie und Ökologie“. Im Mittelpunkt steht dabei die Frage, mit welchen Mitteln es dem modernen Menschen gelingen kann, eine Wiedereinführung der von ihm externalisierten Effekte vorzunehmen, um den Umweltfolgen seines intentionalen Entscheidens gerecht zu werden. Einerseits wird hierzu auf die Systemtheorie Niklas Luhmanns rekurriert, deren kritische Analysen verfolgt und hinsichtlich dieser Frage ausgewertet werden. Zum anderen wird auf Ansätze der Umweltökonomie eingegangen, um die Fragestellung auch andersherum anzugehen. Der Beitrag schließt seine Überlegungen mit einem Statement über „CoEvolution und reflexive Modernisierung“. Vor dem Hintergrund beider Ansätze wird der Prozess einer allmählichen öffentlichen Bewusstwerdung, dass die Ökologie nicht nur eine elementare, im gesellschaftlichen Umgang zu lösende (Gestaltungs-)Aufgabe darstellt, sondern gerade hierin die kulturelle und sozio-ökonomische, als Kennzeichen der derzeitigen historischen Schwelle identifiziert. Im Zusammenhang damit wird ein wachsender Bedarf nicht nur an „Ethik“ und „Vernunft“ selbst, sondern auch an ihrer Problematisierung im öffentlichen Diskurs vermutet.
\end{abstract}

Schlüsselbegriffe: philosophische Gesellschaftstheorie, Verhältnis von Ökonomie und Ökologie, azentrische Umweltethik, Rationalität.

\section{SELBSTREFERENZ UND UMWELTETHIK}

Moral stellt für Niklas Luhmann ein soziales Regulativ dar, welches Handlungen über den binären Code gut / schlecht sanktioniert. ${ }^{2}$ Dadurch erzeugt sie ihre eigene Paradoxie, da eine moralische Konditionierung selbst jeweils sowohl gute als eben auch schlechte Folgen haben kann, die Moral auf sich selbst angewandt also ihre eigene Unmoralität feststellen müsste. ${ }^{3}$ Die Ethik stellt demgegenüber eine Reflexionstheorie der Moral dar,

\footnotetext{
${ }^{1}$ Prof. Dr. habil. Andreas Metzner-Szigeth, Hochschule Bochum, Lennershofstraße 140, 44801 Bochum, mail: andreas.metzner-szigeth@hs-bochum.de

${ }^{2}$ Vgl. N. Luhmann, Ökologische Kommunikation, Opladen 1986, S. 259ff.

${ }^{3}$ Luhmann nimmt hier Max Webers Kritik einer Gesinnungsethik auf, die keine Verantwortung für ihre Folgen übernimmt (vgl. M. Weber, Der Beruf zur Politik, [In:] ders., Soziologie, Universalgeschichtliche Analysen, Politik. Aufsatzsammlung, Hrsg. u. erl. v. J. Winkelmann, 5. Aufl., Stuttgart 1973, S. 167-185), und rearran-
} 
die der Entparadoxierung der moralischen Paradoxie verpflichtet ist; sie stellt sich daher Ersatzprobleme wie z.B. den „kategorischen Imperativ“.

Diese Ausführungen rekurrieren freilich nicht auf die Unterscheidung zwischen moralischem Handeln im Sinne einer verordneten und sanktionierten Moral und solchem, welches aus freiem Willen und Einsicht in die ethische Problematik eines Zusammenhangs erfolgt. ${ }^{4}$ Nichtsdestoweniger ist mit Luhmann anzunehmen, dass mit der notwendigen Theoretizität einer Reflexionsethik die Chancen ihrer praktischen Wirksamkeit sinken.

Hinzu tritt, dass es im Falle von Sozial- und Umweltethik um eine soziale Regulierung von einerseits der Behandlung von Menschen durch Menschen und andererseits der Behandlung von Umwelt durch Menschen geht. Im ersteren Fall ist die Wirksamkeit der Sanktionierung durch Achtung / Missachtung zumindest im Prinzip gesichert, da die innersoziale Komplexität unter den Bedingungen doppelter Kontingenz für eine moralische Konditionierung offen steht. Im zweiten Fall besteht das Dilemma, dass Menschen die Umwelt vor Menschen schützen sollen, die Umwelt selbst aber keine moralischen Sanktionen verhängen kann. ${ }^{5}$

Hinsichtlich der Frage nach ihrer effektiven Wirksamkeit ist daher die Unterscheidung von Moral und Ethik weniger bedeutsam als die Differenz von Sozial- und Umweltethik, deren tieferliegendes Problem auch Luhmann - allerdings nur anreißend - angesprochen hat: Zwangsläufig taucht für die Umweltethik das Problem auf, dass hier die Subjekte der Moral nicht mehr gleich den moralischen Objekten - also Menschen - sind. Diese Inkongruenz ist, wie sich zeigen lässt, durchaus keine rein theoretische, da ökologisch komplexe Probleme zu Konflikten zwischen „Sozial-“ und „Umweltethik“ führen können. Schon das Beispiel einer Eindeichung bei Sturmflutgefahr zeigt die Problematik, da sich der Zielkonflikt auftut, ob die Sicherheit von Menschen (und der für sie günstigen Umwelt samt ihrer Nutzung) für die Ethik bedeutsamer ist als das Leben der Wattflora und Fauna. Die Alternative „Schafweide oder Salzwiese“ mag simpel klingen, zeigt aber das generelle Problem, dass man sich entscheiden muss, auf welcher Grundlage (sozial- oder umweltreferenziell; anthropozentrisch oder nicht-anthropozentrisch) man seine Bewertung durchführen will. Steigt die Komplexität der Problematik, wird die Ethik darüber hinaus zunehmend von einer wissenschaftlichen Analyse abhängig, die als Unterbau einer ethischen

giert die Differenz von Gesinnungs- und Verantwortungsethik in systemtheoretischer Form; vgl. auch A. Metzner-Szigeth, Zwischen Systemkomplexität und Akteursverantwortung, [In:] Technik - System - Verantwortung, Hrsg. K. Kornwachs, Münster u. a. 2004, S. 391-409.

${ }^{4}$ Grundlegend ist hier die von Kant eingeführte Differenz von Heteronomie, also dem Anspruch, dem Sittengesetz gehorchen zu müssen wie einem äußeren Souverän, und Autonomie, also dem Anspruch, einem selbstbestimmten Sittengesetz als innerem Souverän zu folgen. Vgl. I. Kant, Grundlegung zur Metaphysik der Sitten (Erstauflage: Riga 1785), [In:] I. Kant, Werkausgabe, Bd. VII, Hrsg. v. W. Weischedel, Frankfurt a. M. 1982, S. 33ff. Der kategorische Imperativ ist nun der Versuch einer Verbindung des Moments der Allgemeingültigkeit (aus ersterem Begriff) mit dem der individuellen Begründung aus freiem Willen (nach dem zweiten Begriff).

${ }^{5}$ Schleichende Umweltveränderungen oder katastrophische Ereignisse können der Natur als Subjekt zugeschrieben werden, so als ob die Natur auf moralisches Fehlverhalten der Gesellschaft reagieren würde. Als „Rache der Natur" taucht diese Denkfigur in appellativen Bearbeitungen der Umweltproblematik immer wieder auf, scheint aber ein viel älteres Motiv zu sein - man denke nur an die Sintflut. 
Entscheidung diese aber nicht von diesem Dilemma entbinden kann. ${ }^{6}$ In der Öffentlichkeit tritt es wiederum um so schärfer zu Tage, wie (möglicherweise) Betroffene, als Subjekte der Ethik (und damit gleichzeitig originäre Objekte derselben), ihre Ängste artikulieren, während die Natur als Objekt der Umwelt-Ethik ,stumm“ bleibt.

Wie geht die Ethik nun mit dieser Konstitutionsproblematik um? Eine der möglichen Reaktionen darauf ist die Überleitungskonstruktion, die ethisches Handeln gegenüber der natürlichen Umwelt als Erfordernis für das Leben künftiger Generationen begründet. Die zeitliche Dimension wird hier ausgeweitet, um ein aktuelles ethisches Handeln nun doch auf zukünftige Menschen als Objekte der Ethik richten zu können. Dieser Möglichkeit

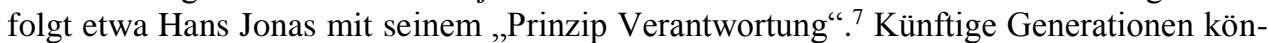
nen sich aber erst in der Zukunft (selbst) artikulieren, nicht aber in der Gegenwart des ethischen Reflektierens vor dem Hintergrund anstehender Entscheidungsprozesse. Wenn nun andere es stellvertretend für sie tun, ist dieses Vorgehen allerdings wieder äquivalent mit einem stellvertretenden Eintreten für die Natur. Gegen wohlgemeinte aber möglicherweise fehlgeleitete Empfehlungen können sich zukünftige Generationen oder die Umwelt ebenso wenig zur Wehr setzen, wie gegen eine Inanspruchnahme der durch Rekurs auf sie möglichen Handlungslegitimation durch und im Interesse der Menschen des hier und heute.

Eine andere Möglichkeit zur Lösung dieses Dilemmas liegt in der sachlichen Dimension, besteht in einer Ausweitung des Umfangs der (Bezugs-)Objekte, auf die sich ethisches Handeln richtet. So unterscheidet etwa Klaus Michael Meyer-Abich „Acht Formen von Rücksichtnahme in der Ethik“, angefangen von einer egozentrischen über eine anthropozentrische bis hin zu einer allumfassenden physiozentrischen Ethik. ${ }^{8}$ Die Deanthropozentrierung erfolgt hierbei in einer Ebenendifferenzierung, die additiv den Umfang des Bereichs, auf den sich ethisches Handeln richtet, erweitert, allerdings ohne dass sich an

\footnotetext{
${ }^{6}$ Einerseits bezieht sich das auf komplexe sozio-ökologische Zusammenhänge. Ein Beispiel dafür wäre etwa eine auf kleinstem Niveau betriebene Weidewirtschaft in einer semiariden Region dieser Welt. Ein forcierter Brunnenbau würde zwar die Ernährungslage verbessern können, aber die nun größeren Herden würden das ihrige zur Desertifikation tun. Vgl. L. Timberlake, Krisenkontinent Afrika - Der Umwelt-Bankrott. Ursachen und Abwendung, Wuppertal 1990. Eine einfach auf Werten, Normen oder idealistischen Motivationen basierende ethische Entscheidung über zu ergreifende Maßnahmen wird hier kaum besser sein können als eine Systemanalyse, die verschiedene Varianten kalkuliert und so eine Strategie zur Optimierung herausarbeiten will, wiewohl letztere lediglich utilitaristisch orientiert ist. Andererseits ist die ethische Behandlung komplexer Technologien, etwa der Gentechnologie, derart massiv an das Niveau fachwissenschaftlicher Theoretizität gebunden, dass ein solcher Diskurs von Ethik und Technik schnell zu einer Diskussion über verschiedene Anwendungsfelder und deren Chancen und Risiken wird. Vgl. Chancen und Risiken der Gentechnologie, Hrsg. Deutscher Bundestag, Der Bericht der Enquete-Kommission des 10. Deutschen. Bundestages, Bonn 1987. Beides macht klar: Der ethische Diskurs verschiebt sich in zunehmendem Maße weg von der Frage, „wie“ eigentlich gehandelt werden soll, hin zu dem Problem, „dass“ die auf der Basis des möglichen Wissens über einen Zusammenhang optimale Entscheidung auch getroffen und getragen wird und nicht etwa eine bequemere, kurzsichtigere oder partikularen Interessen folgende.

${ }^{7}$ Vgl. H. Jonas, Das Prinzip Verantwortung. Versuch einer Ethik für die technologische Zivilisation, Frankfurt a. M. 1987.

${ }^{8}$ Vgl. K.M. Meyer-Abich, Wege zum Frieden mit der Natur. Praktische Naturphilosophie für die Umweltpolitik, München 1986, S. 22.
} 
den Subjekten ethischen Handelns etwas ändert. Belegt wird diese Position mit dem Übergang vom Anthropozentrismus zum Physiozentrismus in der Ethik. ${ }^{9}$

Die gesellschaftliche Natur des Menschen kann von den (Sozial-)Wissenschaften genauso wenig hinterschritten werden, wie die (Umwelt-)Wissenschaften die biologische Natur des Menschen nicht hinterschreiten können. Deutlich wird dies in besonderem Maße in den ,integrativen“ Disziplinen Soziologie und Ökologie. Indem die Ökologie von der Natur handelt, zielt sie auf den Menschen, zielt sie auf die menschliche Lebensweise hinsichtlich ihrer existentiellen Grundlagen, reflektiert sie seine Position und seine Aktivität in der Umwelt. Indem die Soziologie von der Gesellschaft handelt, tut sie ein gleiches, nämlich zielend auf die menschliche Lebensweise gesellschaftliche Selbstorganisationsprozesse erschließen.

Der Mensch als Träger dieses Reflexions- und Handlungszusammenhangs nimmt immer (notwendig) eine ,,anthropozentrische“ Perspektive ein, da er keine andere Möglichkeit hat. Standort der klassischen Naturwissenschaften, der immer noch vorherrschenden naturwissenschaftlichen Denkweise, ist aber der sozusagen säkularisierte ,göttliche Standpunkt" als Subjekt über den Dingen, unvermittelt den Objekten seiner Erkenntnis gegenüberstehend, entsprechend einer Zentrierung der Natur- und Gesellschaftswissenschaften auf das bürgerlich isolierte denkende Subjekt. Die klarer formulierte Perspektive liegt daher in der Ablösung des subjektzentrierten Weltbildes durch ein „,vitalozentrisches ${ }^{\text {“10 }}$, die vermittelt über eine Neubestimmung der Subjektivität des Menschen bezogen auf seine Identität als bewusstes und lebendiges, biotisches und soziales Wesen erfolgt, und geeignet ist, Subjekt und Objekt nicht mehr als unvermittelten Gegensatz zu betrachten, sondern die Beziehungen des Menschen zu anderen Teilen der Natur in ihrer Relativität zu sehen.

Problematisch an der vorherrschenden Form der Ethik-Debatte ist erstens die Tendenz zur Individualisierung. Sie schreibt einzelnen Individuen Verantwortungen zu, die sie allein nicht tragen können, da sie in sozio-ökonomische Handlungssysteme eingebunden agieren. Problematisch ist zweitens die Fixierung auf neuartige Technologien. Notwendig erscheint demgegenüber erstens eine Zentrierung der Diskussion auf demokratische Prozesse der Entscheidungsfindung über Entwicklung und Einsatz alter wie neuer oder ,al-

\footnotetext{
${ }^{9}$ Zur Kontroverse zwischen diesen Perspektiven einer ökologischen Ethik vgl. etwa Ökologie und Ethik, Hrsg. D. Birnbacher, Stuttgart 1986. Bei Meyer-Abich fällt auf, dass er die Problematik der Verbindung zwischen der ethischen Handlungsfähigkeit des Subjektes und seiner anthropozentrischen Perspektive kaum tangiert und letztere schlicht mit einer besitzindividualistisch-materialistischen Einstellung identifiziert. Demgegenüber muss man sagen, dass auch die Idee einer Solidargemeinschaft der ganzen Schöpfung angesichts ihrer ,kosmischen Leidensgeschichte“ (vgl. K.M. Meyer-Abich, Wege zum Frieden mit der Natur..., S. 190ff.) natürlich einer anthropozentrischen Projektion aufruht. Das Problem, dass ethische Normen und Werte ihre Begründung nur in sozialer Verständigung und Erkenntnis finden können, gleichwohl aber die ganze Natur betreffen sollen, wird hier religiös überkompensiert, da klar ist, dass sich aus der Naturordnung als solcher Werte und Normen nicht einfach ableiten lassen können.

${ }^{10}$ Der Begriff des „Physiozentrischen“ kann die Konnotation eines deterministischen Zusammenhangs erzeugen, indem er nur in Richtung auf Physis, die Materie als Gegenstand und Produkt orientiert; es geht aber nicht um eine Ansammlung von Dingen, die mehr oder weniger determiniert naturgesetzlich zusammenhängen, sondern um den allgemeinen Lebenszusammenhang auf diesem Globus, der Ursprung und Grundlage unserer Existenz ist, dem wir einen Sinn oder Wert zumessen, wobei wir die Natur nicht als Menge von Objekten, sondern als schöpferischen Prozess sehen, dessen Elemente auch wir selbst sind.
} 
ternativer" Technologien, auch um Verantwortungen kollektiv, d. h. gemeinsam tragen zu können, und zweitens die Thematisierung des prekären Zusammenhanges von Technologieentwicklung, der Struktur wissenschaftlicher Forschung und deren ökonomischer Initiierung, Durchsetzung und Anwendung, welcher geeignet ist, das Primat einer demokratischen Politik zu unterlaufen.

Festhalten lässt sich, dass Luhmanns Kritik an der Öko-Ethik insofern plausibel ist, als er auf die Dimension der Gesamtgesellschaft aufmerksam macht, die die entscheidende Einheit bezüglich der Ökologiefrage darstellt, die Ethik aber - neben Tendenzen zur Individualisierung - kein Konzept der Vermittlung von Gesellschaft und Natur hat, auf dessen Basis sie das Problem von Anthropozentrik oder Nicht-Anthropozentrik hinreichend wirkungsvoll lösen könnte. Mit Luhmann lässt sich daher einsehen, dass eine Fixierung der Umweltdebatte auf Ethik problematisch wäre, denn: „Wenn irgendwo, stellt jedoch in der ökologischen Kommunikation die Gesellschaft sich selbst in Frage; und es ist nicht einzusehen, wie die Ethik davon dispensiert und als Notanker mit festem Grund bereitgehalten werden könnte“. ${ }^{11}$

Einzuräumen bleibt allerdings, dass es auch keine Gesellschaftstheorie (und schon gar nicht eine, die das Verhältnis zur Natur mit verarbeitet) geben kann, die ohne ein bestimmtes Menschenbild auskommen könnte, das nolens volens mit (wenn schon nicht unbedingt explizierten, so doch immerhin impliziten) ethischen Grundlegungen verbunden ist.

Im Vergleich zu den Bemühungen der Ethik ist zu sagen, dass Luhmanns eigene Theorie dieser gegenüber zwar kritikfähig ist, aber selbst hinsichtlich einer konsequenten Reflexion zur Aufgreifung und Lösung der Ökologiefrage nicht weitreichend genug ansetzt. $^{12}$

Für eine sozio-ökologisch orientierte Theoriebildung ist es demgegenüber von entscheidender Wichtigkeit, die ethische Orientierung mit der unerlässlichen Frage nach gesellschaftlichen Organisationsformen zu verbinden, die die Entfremdung von Mensch und Natur aufheben können. Resümierend lässt sich einbehalten, dass das Verhältnis von erstens einer normativ-ethischen Thematisierung mit dem Ziel einer normenregulierten Regelung, zweitens einer handlungsleitenden kommunikativen Vernunft mit dem Ziel konsensueller Verständigung, und drittens einer in gesellschaftlichen Organisationen zu implementierenden Vernunft mit dem Ziel sozial- und umweltverträglicher Regulierung die alles entscheidende Vernetzung einer ökologischen Umgestaltung darstellt.

\footnotetext{
${ }^{11}$ N. Luhmann, Ökologische Kommunikation..., S. 265.

${ }^{12}$ Dies betrifft nicht zuletzt die ethischen Implikationen ihres eigenen Welt-, Menschen- und Naturbildes, die (mangels Offenlegung) nicht intensiv und selbstkritisch genug verarbeitet werden, um eine wirklich weitreichende Kritikfähigkeit gegenüber konkurrierenden Untersuchungs- und Deutungsangeboten aufbauen zu können. Dies hat seinerseits zur Folge, die Gesellschaft insgesamt von Verantwortung zu entlasten, da sie gar nicht anders kann, als der Eigenlogik von selbstreferentiellen Funktionssystemen zu folgen, weil diese so gedacht sind, als ob sie aus evolutionären Prozessen heraus entstanden seien, die von menschlichen Individuen kaum zu beeinflussen und daher auch kaum zu verbessern sind.
} 


\section{AZENTRIZITÄT UND ÖKOLOGISCHE VERNUNFT}

Vernunft ist Luhmann folgend als Selbstreferenz des Systems, als „Systemrationalität“ zu denken. Diese kann sich, folgert er, nur auf das Operieren des Systems selbst beziehen und nicht auf seine Umwelt. „Ökologische Vernunft" kann daher nur in einer systemeigenen Kontrolle der Rückwirkungen der Umwelt auf die Gesellschaft bestehen. Diese ist aber azentrisch aufgebaut, insofern sie in funktionale Subsysteme differenziert ist. Sie unterliegt somit der jeweiligen Eigenlogik der gesellschaftlichen Subsysteme. Im Weiteren ist „ökologische Vernunft“ nach Luhmann also als je subsystemspezifische Anstrengung zu verstehen, mögliche Veränderungen der eigenen Systemumwelt zu kontrollieren und zu kompensieren. Er schließt, dass in diesem Sinne „ökologische Rationalität erreicht (wäre), wenn die Gesellschaft die Rückwirkungen ihrer Auswirkungen auf die Umwelt auf sich selbst in Rechnung stellen könnte. Für jedes Funktionssystem in der Gesellschaft wäre dieses Prinzip mit entsprechender Systemreferenz zu reformulieren, wobei zu beachten wäre, dass es keine Aggregation solcher Systemrationalitäten geben kann, weil jedes Funktionssystem nur die Eigenrationalität kalkuliert und die Gesellschaft im übrigen als Umwelt behandelt“. ${ }^{13}$ Er begründet dies durch die theoretische Überlegung, dass Systemrationalität erreicht ist, wenn ein System seine System-Umwelt-Differenz in sein Prozessieren wiedereinführt und sich nicht an eigener Identität, sondern an Differenz orientiert.

Gegenüber dieser - zunächst einfach nur einleuchtenden - Denkfigur sind folgende drei Einwände in Erwägung zu ziehen:

(1) Sie ist nicht hinreichend, da sie es unterlässt zu spezifizieren, wie überhaupt - angesichts der (nur) selektiven Perzeptions- und partikular codierten Beurteilungs-Fähigkeit des je fokalen Sozialsystems - diese Rückwirkungen angemessen „,in Rechnung gestellt“. d. h. wahrgenommen und bewertet werden können. Im weiteren bleibt daher unklar, welche Konsequenzen aus einer Situation der (nur) selektiven Perzeptions- und partikular codierten Beurteilungs-Fähigkeiten der Sozialsysteme zu ziehen sind, was nötige Fortentwicklungen oder Korrekturen ihrer eigenlogischen Operationsweise betrifft.

(2) Sie enthält das immanente Problem, dass diese Rationalitätsdefinition für alle System-Umwelt-Verhältnisse gilt, also sowohl für das Verhältnis sozialer Systeme zu anderen sozialen Systemen in ihren jeweiligen Umwelten, wie auch für das Verhältnis psychischer und sozialer Systeme und eben auch für das Verhältnis zur ökologischen Umwelt. Dies impliziert, dass ein soziales System zwischen drei verschiedenen Umwelt(form)en unterscheiden können müsste; es gibt bei Luhmann aber nur den Begriff der einen systemrelativen Umwelt ${ }^{14}$, d. h. es erfolgt keine Qualifizierung der jeweiligen Umweltreferenzen des Systems auf der Basis realer Wechselwirkungen. ${ }^{15}$

\footnotetext{
${ }^{13}$ Ibidem, S. 247.

${ }^{14}$ Ibidem.

${ }^{15}$ Das so gefasste System-Umwelt-Verhältnis führt zu einer „Exteriorisierung“ des Ökologischen (vgl. A. Metzner, Probleme sozio-ökologischer Systemtheorie. Natur und Gesellschaft in der Soziologie Luhmanns, Opladen 1993, S. 169ff.), welches nur semantisch, als (gesellschaftsinterne) „Umwelt“ sozialer Systeme von Bedeutung bleibt. Für die „Menschen“, die in dieser Konzeption als duale Wesen erscheinen, zusammengesetzt aus psychischen und physischen Systemen, gilt etwas Ähnliches, insofern sie in der Umwelt sozialer Systeme ange-
} 
(3) Sie wirft das Problem auf, dass die Frage nach der Vernunft im Verhalten zueinander damit konsequent und ausschließlich auf das Thema Selbsterhaltung des Systems fixiert wird. In diesem Sinne verhält sich ein Chemiekonzern, der FluorChlor-Kohlenwasserstoffe (FCKW) produziert, solange rational, wie diese Produktion nicht negativ auf den Konzern selbst zurückwirkt. Dies mag für die Eigenlogik der ökonomischen Entscheidungsprozesse durchaus zutreffend sein, und es ist nicht unwichtig, dies zu bemerken. Innerhalb der oben angegebenen Rationalitätsbestimmung gibt es aber theoretisch keine denkmögliche Kritik an der Unvernünftigkeit dieser Praxis. Die auch faktisch dennoch wirksame Kritik daran, hat in der Theorie also kein Äquivalent, weil in dieser die Unvernünftigkeit eines Systems sozusagen nur intern erfasst und korrigiert werden kann, nicht aber von außen an dieses herangetragen und Korrekturen notfalls auch erzwungen werden können. ${ }^{16}$

Vor diesem Hintergrund fällt es leicht, den Niederschlag der konzeptionellen Restriktionen zu erkennen, der in der folgenden Aussage Luhmanns offenbar wird: „Der Problemaufriss der Rationalität besagt nicht, dass die Gesellschaft Probleme dieses Formats lösen müsste, um ihr Überleben zu sichern. Fürs Überleben genügt Evolution “ ${ }^{17}$ Einzuwenden ist dagegen zunächst, dass eine heute noch ernstzunehmende Theorie der Evolution diese weder im Sinne eines Fortschritts noch als Garanten des Überlebens verstehen kann. Für Gesellschaften mit einer ökonomischen Basis, die nicht durch die Anwendung einer modernen wissenschaftlichen Rationalität revolutioniert worden ist, kann man annehmen, dass die Integrationskraft mythischer - Natur und Gesellschaft verbindender Weltbilder und daraus abgeleiteter regulativer Normensysteme hinreichend ist, um einen vernünftigen Umgang der Gesellschaft mit der Natur zu gewährleisten. Dann sind in der Tat derartige wissenschaftliche Anstrengungen nicht nötig, und man wird im Großen und Ganzen dem „natürlichen“ Lauf der Dinge Vertrauen schenken können. Für eine soziokulturelle Entwicklung wie die, die die modernen Industriegesellschaften geschaffen hat, ergibt sich aber, dass eine weitere Entwicklung wie bisher die instrumentelle Vernunft und technische Verfügbarkeit über partikulare Ausschnitte von Natur und Gesellschaft immer weiter steigern dürfte, dies aber höchstens zur Beschleunigung der Prozesse ökologischer Selbstdestruktion führt, wenn dem nicht auf entsprechender Ebene eine gesellschaftliche vernünftige Regelung der Austauschbeziehungen an die Seite gestellt wird.

Was sich mit Luhmann einsehen lässt ist, dass Appelle an eine „ökologische Vernunft“ - die z.B. den vernünftigen Gebrauch nicht erneuerbarer Ressourcen gewährleisten sollen - zu kurz gegriffen sind, denn die Frage ökologischer Vernunft muss - um wirksam werden zu können - auf der Ebene gesellschaftlicher Organisationsformen diskutiert und

siedelt werden; die psychischen Systeme werden dabei aber immerhin als mit den sozialen Systemen verkoppelte Entitäten gedacht, weil beide Sinn prozessieren.

${ }^{16}$ Festhalten lässt sich, dass hier ohne Abstriche ökologisches Denken ökonomischer Rationalität untergeordnet wird. Nur insoweit es gelingt, ökologische Erfordernisse in die Sprache der wirtschaftlichen Systemlogik einzufügen, können diese Erfolg haben. Theoretisch und praktisch ist Ökologie aber der umfassendere Begriff, der umfassendere reale Zusammenhang. Wenn nun ökonomische Aktivitäten zwar ökologisch destruktiv, aber trotzdem ökonomisch erfolgreich sind, ist damit klar, dass die rezenten Kriterien ökonomischen Erfolges, die solche „Fehlallokationen“ nicht nur zulassen, sondern geradezu erzwingen, nicht nur ökologisch irrationale sind, sondern damit auch als ökonomische Indikatoren produzierten Wohlstands auf Dauer nicht zu halten sind.

${ }^{17}$ N. Luhmann, Soziale Systeme. Grundriß einer allgemeinen Theorie, Frankfurt a. M. 1984, S. 645. 
gelöst werden. Es reicht weder der „starke Staat“, dem die Freiheit zugeschrieben wird, etwas gegen die zunehmende Umweltverschmutzung zu tun, da ihm erstens keine ökonomischen Verwertungsinteressen unterstellt werden, und zweitens er allein eine volkswirtschaftliche Gesamtperspektive einnehmen kann, noch die „öko-soziale Marktwirtschaft“, von der erhofft wird, sie könnte die umweltdestruktiven Potentiale der kapitalistischen Wirtschaftsweise hinreichend zügeln. Mit Luhmann lässt sich sagen, dass eine ökologische Wende alle gesellschaftlichen Teilbereiche und Subsysteme erfassen müsste, und gegen Luhmann wird man sagen müssen, dass es dabei um eine Systemveränderung gehen muss, wenn sich die „ökologische Kommunikation“ nicht in nur temporären Anpassungsreaktionen niederschlagen und in nutzlosen Ausweichmanövern erschöpfen soll.

Für ein adäquates Problemverständnis und ein hinreichend ausgedehnten Vernünftigkeitsanspruch sind darüber hinaus drei Komplexe wichtig:

(1) Das Problem der zeitlichen Streckung von sozio-ökonomischer Auslösung, ökologischen Effekten, deren Rückwirkung auf die Gesellschaft, der Wahrnehmung dieser Rückwirkungen und schließlich der Reaktion darauf. In Anbetracht dieser Sequenz dürfte es zu spät sein, wenn ein ,System seine Einwirkungen auf die Umwelt an den Rückwirkungen auf es selbst kontrollieren muss" ${ }^{\text {"18 }}$, um sich ökologisch rational zu verhalten. Zumindest wäre einzubeziehen, dass gegenüber Rückwirkungen nicht nur reaktiv, sondern antizipativ (re)agiert werden muss. Dies hängt natürlich mit dem verwertbaren Wissensstand zusammen, beinhaltet auch prinzipielle Unsicherheiten, ist vor allem aber problematisch, da die Rückwirkungen nicht unbedingt den Auslöser treffen, sondern andere - im Falle eines Chemieunfalls in Basel also vor allem die niederländischen Wasserwerke und deren Kunden, von den Fischen, den Fischern und etlichen anderen ganz zu schweigen. Daher muss, um hier überhaupt Problemlösungen anzugehen, ein handlungsfähiges gesellschaftliches „Subjekt“ vorhanden sein, welches zwischen Verursachern und Betroffenen koordinieren und Maßnahmen durchsetzen kann, was auf das Primat einer ,,proaktiven“ Politik verweist.

(2) Die Beziehungen der Gesellschaft zur ökologischen Umwelt müssen im Rahmen der Einbettung des gesellschaftlichen Systems in das ökologische System gesehen werden, d. h. die Frage ökologischer Rationalität muss darauf bezogen werden. Die rein selbstbezügliche Perspektive einer gesellschaftlichen Systemrationalität ist da zu wenig und beruht auf der unhinterfragten Prämisse, dass die erkennenden Subjekte ausschließlich an der Gesellschaft partizipierende Elemente sind und sich (selbst) auch nur als solche verstehen können. Sie können sich (selbst) aber durchaus auch als Elemente ökologischer Systeme sehen und in diesem Sinne sich selbst samt ihrer sozialen Beziehungen auf ihre Position und ihr Verhalten in ökologischen Systemen reflektieren.

(3) Wenn sich die verschiedenen Systemrationalitäten nicht zu einer gesamtgesellschaftlichen Rationalität addieren lassen können, ist klar, dass sie sich in verschiedenem Ausmaß komplementär oder antagonistisch zueinander verhalten; der Bezugspunkt ökologischer Rationalität könnte aber hier zu einem Zusammenwach-

\footnotetext{
${ }^{18}$ Ibidem, S. 642.
} 
sen - oder zumindest einem wechselseitig gesteigerten Verständnis ihrer jeweiligen operationalen Spezialisierungen - beitragen, da er nicht auf einzelne Sozialsysteme und deren selbsterhaltungsfixierte Eigenlogik zu beschränken ist.

\section{3. ÖKONOMIE UND ÖKOLOGIE}

Die Ökologieproblematik wird von der konventionellen (Umwelt-)Ökonomie mit Hilfe der Differenz von betriebs- und volkswirtschaftlicher Perspektive bearbeitet. ${ }^{19}$ Nutzung und Verschmutzung der Umwelt erscheinen so als ressourcenökonomische Kollektivgutund Allokationsproblematik. Die theoretische Problemlösung gelingt folgerichtig nur unter Auflösung der unterlegten Differenz (von Privatwirtschaft und Allgemeinwohl) und verlangt nach einer betrieblichen Internalisierung der Kosten „externer Effekte“. Das sich derart ergebende „umweltökonomische Optimum“20, als das günstigste Verhältnis der Nutzung von ökologischen Ressourcen gegenüber den volkswirtschaftlichen Kosten der Umweltverschmutzung bzw. den Kosten, die entstehen, wenn man diese vermeiden oder wieder reparieren will, trägt die Konnotation eines Gleichgewichts von Natur und Gesellschaft. Da aber ökologische Parameter in diese Rechnung gar nicht eingehen, ist zu fragen, wie eine Intakterhaltung ökologischer Systeme garantiert werden kann, selbst wenn dieses Kalkül tatsächlich Preise und ökonomisches Handeln bestimmen würde.

Die an Karl Marx orientierte Theoriebildung löst die Problematik hingegen nach der Differenz von Kapital und Arbeit auf. Sie erscheint dann als Interessenswiderspruch hinsichtlich der Aneignung von Werten und damit letztlich der Verwertung von Umweltressourcen. Über die Analogisierung der Verwertungsanalyse der Arbeitskraft mit der Verwertung der Natur, wobei deren Reproduktionskosten im Mittelpunkt stehen, wird eine Strategie submaximaler Nutzung derselben formuliert, welche im Interesse der Gesamtgesellschaft günstiger erscheint. ${ }^{21}$ Dabei wird, wie in der Umweltökonomie fast generell, die gesellschaftsinterne Differenz aufgelöst, welche zuvor durch die Theorie begründet wurde. Nur auf diese Weise kann das problematische Verhältnis von Ökologie und Gesellschaft so dargestellt werden, dass es innerhalb der Theorie als gelöst erscheint.

Die ökologische Dimension ist damit in beiden Fällen zwar keine marginale Bezugsgröße mehr, bleibt aber den herrschenden sozialwissenschaftlichen Denktraditionen insoweit verhaftet, als sie eine residuale Kategorie bleibt und folglich nicht zu einem konstitutiven Teil der Theorie wird. Statt dessen wird die Umweltproblematik in eine schon vorgängig ausgebildete und auf anderen Feldern „bewährte“ Theoriearchitektur eingegliedert, wo angesichts der Tiefe und der Fülle neuer Fragestellungen und brennender Probleme, wie sie die ökologische Krise aufgeworfen hat, eine weitreichende Neukonstruktion notwendig wäre.

Die Praxisprobleme, die durch die auf uns eindringenden Veränderungen unserer Umwelt, sowie durch die daraus folgende Notwendigkeit einer ökologischen Umgestal-

\footnotetext{
${ }^{19}$ Vgl. W.K. Kapp, Soziale Kosten der Marktwirtschaft, Frankfurt a. M. 1979.

${ }^{20}$ Vgl. etwa L. Wicke, W. Franke, Umweltökonomie. Eine praxisorientierte Einführung, München 1982, S. 17ff., $222 \mathrm{ff}$.

${ }^{21}$ Vgl. hierzu u. a. E. Gärtner, Arbeiterklasse und Ökologie, Frankfurt a. M. 1979; H. Immler, Natur in der ökonomischen Theorie, Opladen 1985; H. Immler, W. Schmied-Kowarzik, Marx und die Naturfrage. Ein Wissenschaftsstreit, Hamburg 1984.
} 
tung der modernen Industriegesellschaft aufgeworfen werden, verlangen nach interdisziplinärer Zusammenarbeit. Es gilt diese durch eine forcierte transdisziplinäre Theoriebildung auszubauen und zu stabilisieren. Genau in diesem Zusammenhang ist festzustellen, dass ohne Beachtung und Assimilation des Erkenntnisstandes der Ökologie und ihrer Modelle auch die „Ökologie der Gesellschaft“ nicht mit der gebotenen Tiefe zu bearbeiten ist.

Anknüpfungspunkt für solche Überlegungen ist das Zentralmodell des ökologischen Systems. Im Milieu der Systemtheorie ist hier in engem Kontakt zu Biologie und Umweltforschung ein heuristisch fruchtbares, methodisch breit angelegtes und formal ausbaufähiges Konzept herangereift. ${ }^{22}$ Mit seiner konzeptionellen Orientierung auf die Ganzheit der Natur und die Verbundenheit ihrer mannigfaltigen Komponenten bietet der Ökosystembegriff einen in die Zukunft weisenden Alternativweg zwischen romantischen Vorstellungen einer beseelten Naturganzheit und einer sich weitgehend auf isolierte Zusammenhänge beschränkenden analytisch-reduktionistischen Naturforschung. Umgekehrt gilt allerdings, dass die ökologische Krise ebensowenig allein mit Hilfe naturalistischer Theorieansätze hinreichend zu erschließen ist. Denn so fehlt dem ökologischen Denken ein gesellschaftstheoretischer Rahmen, der geeignet ist, die Historizität von Veränderungen innerhalb der Entwicklung gesellschaftlicher Systeme mit der Dynamik der Veränderung ökologischer Systeme zu verknüpfen. Gesellschaften sind in ihrer materiellen Existenz Teil der ökologischen Systeme, auch wenn diese häufig - sowohl für die herrschende gesellschaftliche Praxis, wie auch für die daran orientierte sozialwissenschaftliche Analyse - als naturale Umwelt $^{23}$, als außerhalb der Gesellschaft stehende Natur erscheinen.

Folgt man den gerade skizzierten Überlegungen, so wird einsichtig, dass die ökologische Krise strenggenommen weder als Krise der „Umwelt“ noch als solche ,gesellschaftlicher Institutionen“ betrachtet werden kann. ${ }^{24}$ Sie betrifft das Innen und das Außen der Gesellschaft genauso wie das Innen und das Außen ökologischer Systeme. Zusätzlich kompliziert ist dieses Verhältnis dadurch, dass keine reziproken Perspektiven vorliegen, das Außen der Gesellschaft also nicht zwangsläufig deckungsgleich ist mit dem Innen ökologischer Systeme und umgekehrt. Als Kategorien sind Innen und Außen, System und Umwelt, Natur und Gesellschaft symbolisch erzeugte Konstrukte, die aufeinander bezugnehmend definiert werden. Im Kontext verschiedener Denkweisen ergibt sich ein variierender Umfang und eine verschiedene Bedeutung dieser Begriffe. Diese handlungsleiten-

${ }^{22}$ Vgl. u.a. B. Breckling, Naturkonzepte und Paradigmen in der Ökologie. Einige Entwicklungen, Berlin (WZB) 1993.

${ }^{23}$ Die Vielschichtigkeit des Begriffs der Natur macht den Rekurs auf ihre „Natürlichkeit“ problematisch. Immerhin lassen sich zwei mögliche Missverständnisse ausräumen, wenn man statt von ,natürlicher“ von ,naturaler" Umwelt spricht. Erstens: Es bleibt offen, inwieweit die Umwelt durch menschliche Aktivitäten mitgeprägt ist oder inwieweit sie ohne anthropogene Einflüsse geblieben ist. Zweitens: Es bleibt offen, was ein aus der Sicht des Menschen wünschenswerter, anzustrebender Zustand der Umwelt ist, und ob dieser einem ökologisch stabilen, ,gleichgewichtigen“ Zustand der Umwelt entspricht. Da alle menschlichen Gesellschaften durch ihre Kultur geprägt sind, gibt es keinen „Naturzustand“, weder für den Menschen noch seine Gesellschaft. Überall wo Menschen existieren, beeinflussen sie als Naturwesen zwangsläufig ihre Umwelt. „Natürlichkeit“ im Sinne eines vom Menschen unbeeinflussten Zustandes kann für die Ökologie der Gesellschaft also kein Ziel sein. Der Begriff des „Naturalen“ ist hier neutraler, und hält Fragen und Diskussionsbedarf für einen konstruktiven Entscheidungsfindungsprozess offen.

${ }^{24}$ Vgl. U. Beck, Die organisierte Unverantwortlichkeit, Frankfurt a. M. 1988, S. $92 \mathrm{f}$. 
den Denkweisen wiederum, und die mit ihnen gegebenen Modelle des Verhältnisses von System und Umwelt, folgen der gesellschaftlichen Differenzierung, und - in theoretisch systematisierter Form - der Differenzierung wissenschaftlicher Disziplinen. Was die Soziologie als „Außen“ der Gesellschaft betrachten mag, stellt sich für die Ökonomie als ressourcen- und güterwirtschaftliche Ebene der Produktion durchaus als im „Inneren“ ihres gesellschaftswissenschaftlichen Gegenstandsbereichs liegende Größe dar. Für die Ökologie liegen diese Vorgänge wiederum zunächst nicht im Inneren ihres Gegenstandsbereichs, sondern sie registriert sie als anthropogene, von außen erfolgende Einwirkungen auf ökologische Systeme. Für die interdisziplinäre Diskussion und transdisziplinäre Modellbildung ist damit ein beträchtlicher Abstimmungs- und Klärungsbedarf vorgegeben.

\section{CO-EVOLUTION UND REFLEXIVE MODERNISIERUNG}

Die vorgestellten Überlegungen zu den Themen „Selbstreferenz und Umweltethik“, „Azentrizität und ökologische Vernunft“ sowie zum Verhältnis von „Ökonomie und Ökologie“ lassen darauf schließen, dass integrative Konzepte zur Bearbeitung der „Ökologie der Gesellschaft" notwendig sind, zumindest, um das wieder zusammenzufügen, was im Zuge der Entwicklung der vielen einzelnen wissenschaftlichen Disziplinen voneinander geschieden wurde. Zwei Ansätze hierzu sind das Konzept der „Co-Evolution“25 und das der ,reflexiven Modernisierung “26.

Das erste Konzept setzt am Gedanken der Co-Evolution der verschiedenen Populationen einer Biozönose an, die eine immerwährende Dynamik in Richtung einer Optimierung ihres Ökosystems erzeugt. Die Konfigurierung von Materiekreislauf und Energiedurchsatz erfolgt dabei in Richtung der bestmöglichen Nutzung aller verfügbaren Ressourcen. Es vertritt die These, dass der Mensch in seiner gesellschaftlichen Entwicklung aus dieser Co-Evolution freigesetzt bzw. ausgeschert ist. Seine ökologische Potenz entwickelt sich nicht mehr allein auf Basis der organischen Evolution von zufälliger Variation und Selektion, sondern vornehmlich auf Basis einer soziokulturellen Entwicklung durch gerichtete Lernprozesse. Diese schlagen sich in entwickelteren Formen sozialer Kooperation und Arbeitsteilung, effektiveren Organisationen gesellschaftlicher Arbeit und Produktion, leistungsfähigeren Technologien etc. nieder. Die Spezies „homo sapiens“ kann daher in die abiotischen und biotischen Gefüge von Ökosystemen in einer Qualität und einem Maße eingreifen, die keinem anderen Lebewesen möglich ist. Wenn aber die soziokulturelle Evolution des Menschen gleichzeitig mit dem herkömmlichen Muster organischer Co-Evolution bricht, ohne dass die Spezies „Mensch“ damit aus ihrer absoluten

\footnotetext{
${ }^{25}$ vgl. u. a. F. Hinterberger, (Ko?)Evolution von Natur, Kultur und Wirtschaft. Einige modelltheoretische Überlegungen, [In:] Zwischen Entropie und Selbstorganisation. Perspektiven einer ökologischen Ökonomie, Hrsg. F. Beckenbach, H. Diefenbacher, Marburg 1994, S. 317-348, sowie A. Metzner, Offenheit und Geschlossenheit in der Ökologie der Gesellschaft, [In:] Zwischen Entropie und Selbstorganisation. Perspektiven einer ökologischen Ökonomie, Hrsg. F. Beckenbach, H. Diefenbacher, Marburg 1994, S. 349-391, A. Metzner, Constructions of Environmental Issues in Scientific and Public Discourse, [In:] Eco Targets, Goal Functions and Orientors, eds. F. Müller, M. Leupelt, Berlin a. o. 1998, pp. 171-192.

${ }^{26}$ Vgl. u. a. U. Beck, Risikogesellschaft. Auf dem Weg in eine andere Moderne, Frankfurt a. M. 1986 sowie U. Beck, A. Giddens, S. Lash, Reflexive Modernization. Politics, Tradition and Aesthetics in the Modern Social Order, Cambridge 1994.
} 
Einbindung in die Strukturgefüge ökologischer Systeme „,befreit“ wäre, ist zu folgern: Die ökologische Problematik evolutionären „Fortschritts“ der menschlichen Gattung wird zu einer permanent $\mathrm{zu}$ bewältigenden und mit zunehmenden wissenschaftlich-technischen und ökonomisch-sozialorganisatorischen Möglichkeiten auch immer schwieriger zu bewältigenden Aufgabe, da die Komplexität, Mächtigkeit und Tiefe des menschlichen Wirkens innerhalb ökologischer Systeme zunehmen. Damit wächst nicht nur das Risiko- und Rückwirkungspotential, sondern es wird der Möglichkeit nach räumlich entgrenzt und zeitlich ausgedehnt. ${ }^{27}$

Als Kennzeichen der derzeitigen historischen Schwelle darf hierbei, im Anschluss an das zweite Konzept, welches das Phänomen der reflexiven Modernisierung in den Mittelpunkt rückt, der Prozess der allmählichen Bewusstwerdung gelten, dass die Ökologie nicht nur eine elementare, im gesellschaftlichen Umgang zu lösende (Gestaltungs)Aufgabe darstellt, sondern gerade hierin die kulturelle und sozio-ökonomische. ${ }^{28}$ Entwicklungsperspektive der Moderne für sich selbst zum Problem geworden ist. ${ }^{29}$ Im Anschluss daran ist zu vermuten, dass „Ethik“ und „Vernunft“ im öffentlichen Diskurs nicht einfach nur stärker nachgefragt werden, sondern auch ein wachsender Bedarf an ihrer Problematisierung besteht.

\section{LITERATUR}

[1] Beck U., Risikogesellschaft. Auf dem Weg in eine andere Moderne, Frankfurt a. M. 1986.

[2] Beck U., Die organisierte Unverantwortlichkeit, Frankfurt a. M. 1988.

[3] Beck U., Giddens A., Lash S., Reflexive Modernization. Politics, Tradition and Aesthetics in the Modern Social Order, Cambridge 1994.

[4] Breckling B., Naturkonzepte und Paradigmen in der Ökologie. Einige Entwicklungen, Berlin (WZB) 1993.

[5] Chancen und Risiken der Gentechnologie, Hrsg. Deutscher Bundestag, Der Bericht der Enquete-Kommission des 10. Deutschen. Bundestages, Bonn 1987.

[6] Gärtner E., Arbeiterklasse und Ökologie, Frankfurt a. M. 1979.

[7] Hassenpflug D., Sozialökologie. Ein Paradigma, Opladen 1993.

[8] Hinterberger F., (Ko?)Evolution von Natur, Kultur und Wirtschaft. Einige modelltheoretische Überlegungen, [In:] Zwischen Entropie und Selbstorganisation. Perspektiven einer ökologischen Ökonomie, Hrsg. F. Beckenbach, H. Diefenbacher, Marburg 1994, S. 317-348.

[9] Immler H., Natur in der ökonomischen Theorie, Opladen 1985.

[10] Immler H., Schmied-Kowarzik W., Marx und die Naturfrage. Ein Wissenschaftsstreit, Hamburg 1984.

\footnotetext{
${ }^{27}$ Vgl. weiter A. Metzner, Die Tücken der Objekte. Über die Risiken der Gesellschaft und ihre Wirklichkeit, Frankfurt a. M. u. a., A. Metzner-Szigeth, Contradictory Approaches? - On Realism and Constructivism in the Social Sciences Research on Risk, Technology and the Environment, "Futures" 41/2 (2008), pp. 156-170, http://dx.doi.org/10.1016/j.futures.2008.09.017.

${ }^{28}$ Vgl. auch D. Hassenpflug, Sozialökologie. Ein Paradigma, Opladen 1993, S. 158ff.

${ }^{29}$ Vgl. A. Metzner-Szigeth, Kultur \& Technik als Medien menschlicher Selbstverwirklichung - Überlegungen zur philosophischen Anthropologie und Gesellschaftstheorie, [In:] Technik und Kultur - Bedingungs- und Beeinflussungsverhältnisse, Hrsg. G. Banse, A. Grunwald, Karlsruhe, pp. 143-162, http://egora.unimuenster.de/ifs/personen/bindata/20100713_Metzner-Szigeth_Kultur-und-Technik-als-Medien-menschlicherSelbstverwirklichung.pdf.
} 
[11] Jonas H., Das Prinzip Verantwortung. Versuch einer Ethik für die technologische Zivilisation, Frankfurt a. M. 1987.

[12] Kant I., Grundlegung zur Metaphysik der Sitten (Erstauflage: Riga 1785), [In:] ders., Werkausgabe, Bd. VII, Hrsg. v. W. Weischedel, Frankfurt a. M. 1982.

[13] Kapp W.K., Soziale Kosten der Marktwirtschaft, Frankfurt a. M. 1979.

[14] Luhmann N., Soziale Systeme. Grundriß einer allgemeinen Theorie, Frankfurt a. M. 1984.

[15] Luhmann N., Ökologische Kommunikation, Opladen 1986.

[16] Metzner A., Probleme sozio-ökologischer Systemtheorie. Natur und Gesellschaft in der Soziologie Luhmanns, Opladen 1993.

[17] Metzner A., Offenheit und Geschlossenheit in der Ökologie der Gesellschaft, [In:] Zwischen Entropie und Selbstorganisation. Perspektiven einer ökologischen Ökonomie, Hrsg. F. Beckenbach, H. Diefenbacher, Marburg 1994, S. 349-391.

[18] Metzner A., Constructions of Environmental Issues in Scientific and Public Discourse, [In:] Eco Targets, Goal Functions and Orientors, eds. F. Müller, M. Leupelt, Berlin a. o. 1998, pp. 171-192.

[19] Metzner A., Die Tücken der Objekte. Über die Risiken der Gesellschaft und ihre Wirklichkeit, Frankfurt a. M. u. a. 2002.

[20] Metzner-Szigeth A., Zwischen Systemkomplexität und Akteursverantwortung, [In:] TechnikSystem - Verantwortung, Hrsg. K. Kornwachs, Münster u. a. 2004, S. 391-409.

[21] Metzner-Szigeth A., Contradictory Approaches? - On Realism and Constructivism in the Social Sciences Research on Risk, Technology and the Environment, "Futures" 41/2 (2008), pp. 156-170, http://dx.doi.org/10.1016/j.futures.2008.09.017.

[22] Metzner-Szigeth A., Kultur \& Technik als Medien menschlicher Selbstverwirklichung - Überlegungen zur philosophischen Anthropologie und Gesellschaftstheorie, [In:] Technik und Kultur - Bedingungs- und Beeinflussungsverhältnisse, Hrsg. G. Banse, A. Grunwald, Karlsruhe, pp. 143-162, http://egora.uni-muenster.de/ifs/personen/bindata/20100713_MetznerSzigeth_Kultur-und-Technik-als-Medien-menschlicher-Selbstverwirklichung.pdf.

[23] Meyer-Abich K.M., Wege zum Frieden mit der Natur. Praktische Naturphilosophie für die Umweltpolitik, München 1986.

[24] Ökologie und Ethik, Hrsg. D. Birnbacher, Stuttgart 1986.

[25] Timberlake L., Krisenkontinent Afrika - Der Umwelt-Bankrott. Ursachen und Abwendung, Wuppertal 1990.

[26] Weber M., Der Beruf zur Politik, [In:] ders., Soziologie, Universalgeschichtliche Analysen, Politik. Aufsatzsammlung, Hrsg. u. erl. v. J. Winkelmann, 5. Aufl., Stuttgart 1973, S. 167-185.

[27] Wicke L., Franke W., Umweltökonomie. Eine praxisorientierte Einführung, München 1982.

\section{ECOLOGY AND SOCIETY: ABOUT ETHICS AND RATIONALITY AND RATIONALITY IN RELATION BETWEEN SYSTEM AND ENVIRONMENT}

Without an extended approach for our thinking and acting there will be no solution of the environmental problem of modernity. Rationality and ethics are challenged therefore, to enlarge the possibilities of our insight in this problem and our orientation regarding this problem. In order to underline the principal complications that are to be mastered on this way, this paper is working on the themes "Self-Referency and Environmental Ethics", "Acentricity and Ecological Rationality" and on the relationship of "Economy and Ecology". Hereby it will be focused on the question, which media are needed to empower the modern human to arrange a re-entry of the externalized effects, in order to master the environmental consequences of his intentional decisions. On the one hand we will recur on the systems theory of Luhmann, whose critical analyses will be viewed and assessed regarding this question. On the other hand approaches of environmental economy will be considered, 
in order to tackle the question also from the other side. The paper concludes its considerations with a statement about "Co-Evolution and Reflexive Modernization". In the background of both concepts is the process of gradual social awareness that the ecology is not only an elementary task, which in social intercourse can be solved, but the cultural and socio-economic shaping task, identified as characteristic of the current historical threshold. Related to, a growing need not only to "ethics" and "reason" itself, but also to their problematization in public discourse should be expected.

Keywords: philosophical theory of society, relations between economy and ecology, acentric environmental ethics, rationality.

\section{DOI: 10.7862/rz.2014.mmr.32}

Tekst złożono w redakcji: wrzesień 2014

Przyjęto do druku: październik 2014 